\title{
Recambios de artroplastía de rodilla en el Uruguay en un período de 10 años: supervivencia y resultados
}

\author{
Knee arthroplasty revision in Uruguay over a 10-year period: survival and results \\ Pintos-Demichelis B,* Pache S,* Francescoli L ${ }^{\ddagger}$ \\ Universidad de la República.
}

RESUMEN. Introducción: Según el FNR (Fondo Nacional de Recursos) se ha observado un aumento del número de recambios de ATR (artroplastía total de rodilla) en nuestro país. No hay estudios que evalúen sus resultados. El objetivo del estudio fue analizar la supervivencia, evaluar resultados y analizar la población de los recambios de ATR. Material y métodos: Se realizó un estudio retrospectivo de cohorte observacional y descriptivo de 156 casos de recambio de ATR registrados en el FNR, entre 2004 y 2014. El análisis del porcentaje acumulado de supervivencia se realizó con el método de Kaplan-Meier, utilizando la re-revisión como evento de interés. Se utilizó el puntaje WOMAC, KOOS y SANE para evaluar los resultados funcionales. Resultados: El porcentaje acumulado de supervivencia fue de 85\% a 10 años. Los puntajes promedio fueron: KOOS 61.5, WOMAC 29.8 y SANE 65. Se registraron complicaciones en $25 \%$ de los casos (infección $14.10 \%$ ). El promedio de edad fue de 71 años y $60.9 \%$ de los casos fueron en mujeres. De los recambios, $62.82 \%$ fue de causa aséptica y en la gran mayoría (89.1\%) se utilizó prótesis constreñida. Conclusión: El porcentaje acumulado de supervivencia de los recambios de ATR fue de $86 \%$ a 10 años según el método de Kaplan-Meier. Sólo 51\% de los recambios era funcional a 10 años. Hemos de ser precavidos al interpretar los resultados obtenidos con este método. Se registraron resultados funcionales aceptables comparados con otros registros, aun-
ABSTRACT. Introduction: According to the NRF (National Resource Fund) there has been an increase in the number of TKA (total knee arthroplasty) in our country. There are no studies evaluating their results. The objective of the study was to analyze survival, evaluate results and analyze the population of ATR replacements. Material and methods: A retrospective observational and descriptive cohort study of 156 cases of ATR turnover registered in the NRF was conducted between 2004 and 2014. The analysis of the cumulative percentage of survival was performed with the Kaplan-Meier method, using re-revision as an event of interest. The WOMAC, KOOS and SANE score were used to assess functional outcomes. Results: The cumulative percentage of survival was $85 \%$ at 10 years. The average scores were: KOOS 61.5, WOMAC 29.8 and SANE 65. Complications were recorded in $25 \%$ of cases (infection $14.10 \%$ ). The average age was 71 years and $60.9 \%$ of the cases were in women. $62.82 \%$ of the replacements were of aseptic cause and in the vast majority (89.1\%) constricted prostheses were used. Conclusion: The cumulative percentage of survival of revision TKA was $86 \%$ at 10 years according to the Kaplan-Meier method. Only $51 \%$ of revisions were functional at 10 years. We must be cautious when interpreting the results obtained with this method. Acceptable functional outcomes were recorded compared to other registries, although patient satisfaction

\section{Nivel de evidencia: IV}

* Médico. Egresado de Traumatología y Ortopedia.

₹ Profesor de la Cátedra de Traumatología y Ortopedia.

Universidad de la República. (UdelaR).

Correspondencia:

Dr. Bruno Pintos-Demichelis

Pedro Campbell Núm. 1407, Montevideo, C.P. 11600, Uruguay.

E-mail: brunopintos86@gmail.com

Recibido: 09-07-2020. Aceptado: 30-06-2021.

Citar como: Pintos-Demichelis B, Pache S, Francescoli L. Recambios de artroplastía de rodilla en el Uruguay en un período de 10 años: supervivencia y resultados. Acta Ortop Mex. 2021; 35(3): 276-281. https://dx.doi.org/10.35366/102367

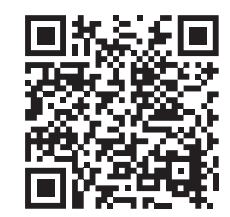


que el grado de satisfacción de los pacientes fue bajo en más de un tercio de los casos y existió un alto porcentaje de complicaciones. Se observó una tendencia hacia el aumento de la tasa de recambio entre 2004 (1.4\%) y 2014 (2\%).

Palabras clave: Artroplastía de rodilla de revisión, supervivencia, resultados clínicos.

\section{Introducción}

La artroplastía total de rodilla (ATR) ha aumentado en los últimos años en forma considerable, dada su probada seguridad y efectividad para el tratamiento de la gonartrosis en sus etapas finales. En Estados Unidos, la incidencia de ATR primaria ha aumentado de 51 por cada 100,000 habitantes en 1990, a 215 por cada 100,000 en 2008. ${ }^{1}$ Por otra parte, en Suecia ha aumentado de 115 por cada 100,000 en 2007 a 135 por cada 100,000 en 2011, mientras que en Noruega, de 35 por cada 100,000 en 1999 a 90 por cada 100,000 en 2011. . $3,4,5^{4}$ En nuestro medio el uso de ATR ha aumentado de 904 en 2004 a 2,178 en 2016, según el registro del Fondo Nacional de Recursos (FNR). ${ }^{6}$ Ésta es una institución pública no estatal que brinda cobertura financiera a procedimientos de medicina altamente especializada para toda la población residente en nuestro país.

Con un aumento de la población de adultos mayores y el uso de ATR en pacientes cada vez más jóvenes, el aumento de ATR persistirá, al igual que el volumen de recambios de ATR. ${ }^{78}$ Por ejemplo, en Noruega la razón entre la tasa de revisión y el número total ATR ha aumentado de $6.9 \%$ en 1994 a 8.5\% en $2011 .{ }^{4} \mathrm{Si}$ bien sabemos que la ATR primaria tiene una supervivencia mayor de $95 \%$ a 15 años, poco se sabe sobre la supervivencia de la ATR de revisión, aunque algunos estudios muestran supervivencias bajas de hasta $60 \%$ en menos de 10 años. ${ }^{9.10,11}$

En nuestro medio, si bien el número de recambios anuales de ATR ha ido en aumento en los últimos años, aún no existen estudios sobre la supervivencia de las mismas y la tasa de recambio.

Nuestro objetivo principal fue analizar retrospectivamente la supervivencia de los recambios de ATR realizados en el período comprendido entre 2004 y 2014 en el país, según los registros del FNR. Como objetivo secundario buscamos describir y analizar la población y valorar los resultados funcionales de éstos.

\section{Material y métodos}

Estudio retrospectivo de cohorte observacional y descriptivo de los pacientes operados a través del FNR de recambio de ATR en el período comprendido entre 2004 y 2014 en nuestro país. Se excluyeron otros tipos de revisión como la colocación de espaciadores o retiro parcial de algún componente. En una primera instancia se analizaron los datos was low in more than one third of cases and there was a high percentage of complications. A trend towards an increase in the turnover rate was observed between 2004 (1.4\%) and 2014 (2\%).

Keywords: Revision knee arthroplasty, survivorship, clinical outcomes.

aportados por el FNR desde el punto de vista epidemiológico destacando las siguientes variables: edad, sexo, motivo principal del recambio primario, tipo de prótesis utilizada, mortalidad de los pacientes durante el estudio, comorbilidades y tasa de recambio anual. Los motivos de recambio se dividieron en dos grandes grupos: sépticos o asépticos. A su vez este último grupo se dividió en las siguientes categorías: aflojamiento, desgaste y ruptura (D y R), inestabilidad, fractura y otros. Ninguna de estas categorías es excluyente. Si el motivo de recambio fue séptico en combinación con otras causas, se tomó la primera como la causa principal.

En una segunda etapa se contactó telefónicamente a los pacientes y se aplicó un cuestionario preestablecido para determinar supervivencia, resultado funcional, satisfacción y complicaciones.

El análisis de la supervivencia se realizó con el método de Kaplan-Meier, mientras que el resultado funcional fue estudiado mediante el puntaje de KOOS (Knee Injury and Osteoarthritis Outcome Score), (KOOS en español) y el WOMAC (Western Ontario and McMaster Universities Osteoarthritis Index). ${ }^{12,13}$ A su vez se determinó el puntaje de satisfacción postoperatorio SANE (Single Assessment $\mathrm{Nu}$ meric Evaluation) para cada caso. ${ }^{14}$ Se definió revisión como la remoción, adición o cambio parcial o total del implante por primera vez. «Re-revisión» se definió como la revisión de la revisión de la ATR. «Recambio» se definió como la sustitución del implante por otro por primera vez, mientras que el «re-recambio» fue la sustitución del recambio. Definimos como fracaso a aquellos casos en los que fue necesario una revisión. Las limpiezas quirúrgicas aisladas no cuentan como revisión, re-revisión o fracaso del recambio.

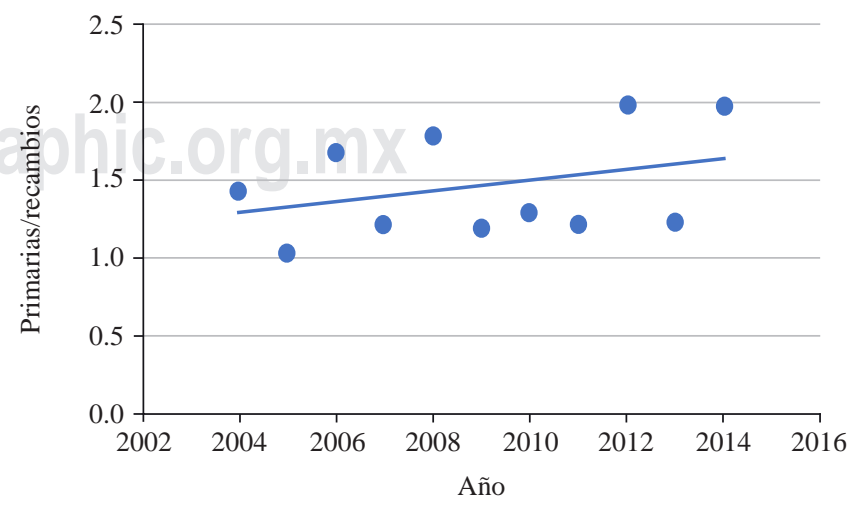

Figura 1: Tasa de recambio de artroplastía total de rodilla. 


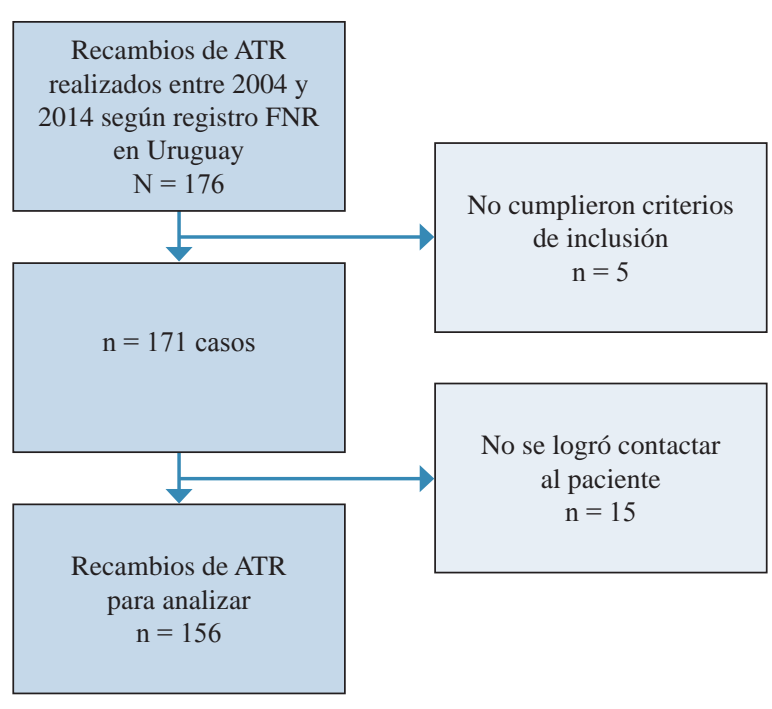

Figura 2: Flujograma de recolección de casos.

ATR $=$ artroplastía total de rodilla, FNR $=$ Fondo Nacional de Recursos.

\section{Resultados}

El FNR nos aportó datos sobre 176 recambios en sus registros. Vale destacar que este número representa el total de recambios realizados en nuestro país en los tres centros especializados existentes. La tasa de recambio de ATR en 2004 fue de 1.4\% y aumentó hasta 2\% en 2014 (Figura 1). De éstos, se descartaron cinco casos por no cumplir los criterios de inclusión (dos resurfacing, un re-recambio y en dos no se realizó el recambio pese a haber llenado el formulario) quedando 171 casos en 169 pacientes. De éstos, logramos comunicarnos con 154 pacientes o familiares, con 156 recambios de ATR (Figura 2). Se obtuvo entonces una pérdida de $8.7 \%$ de los casos. El promedio de edad de estos pacientes al momento de la cirugía fue de 71 años (26-90 años). Durante el período de estudio se registró una mortalidad total de $23.72 \%$, siendo de $5.13,12.82$ y $21.15 \%$ a dos, cinco y 10 años respectivamente. Hubo 81 casos de rodilla derecha y 75 de rodilla izquierda. En cuanto al sexo, 95 (60.9\%) fueron mujeres, mientras que 61 (39.1\%) fueron hombres. Hubo 139 casos (89.1\%) de prótesis de tipo semiconstreñido no abisagrado (TC3, Johnson \& Johnson), 13 (8.33\%) fueron prótesis no constreñidas, una prótesis modular tumoral (0.64\%) y en dos de los casos (1.28\%) no se registraron datos.

En lo que respecta a la indicación registrada por el FNR, 98 casos (62.82\%) fueron por causa aséptica y 58 (37.18\%) casos por causa séptica. Dentro de las asépticas, en nueve casos $(9.28 \%)$ no se especificó la causa, 43 casos $(43.88 \%)$ fueron por aflojamiento aséptico, 25 casos (25.51\%) por DyR, por inestabilidad 17 casos (17.35\%) y cuatro casos (4.08\%) por fractura periprotésica. El seguimiento promedio fue de 6.7 años con un mínimo de 0 y máximo de 14 años.

La estimación de la supervivencia con los intervalos de confianza a $95 \%$ para dos, cinco y 10 años fueron $93 \%$ (89.4; 97.7), 89\% (83.8; 94.3), 85\% (80; 91.6), respecti- vamente (Figura 3). Cabe destacar que a dos, cinco y 10 años existieron 148, 132 y 113 pacientes en riesgo respectivamente. Este porcentaje a 10 años de seguimiento fue de $86.87 \%$ para causa aséptica y $85.96 \%$ para causa séptica, sin diferencia significativa $(\mathrm{p}=0.902)$.

Se registraron 39 complicaciones postoperatorias (25\%), dentro de las cuales hubo 22 casos de infección del sitio quirúrgico, lo cual constituye $14.10 \%$ de los casos. Hubo 11 complicaciones, en las cuales no se realizó reintervención quirúrgica. Dentro de éstas hubo tres infecciones, dos aflojamientos, dos casos de deterioro general severo, una HDA, una fractura periprotésica y un caso de rigidez.

Hubo 28 reintervenciones quirúrgicas (18\% del total de recambios), de las cuales 21 fueron re-revisiones (13.5\%) (Figura 4). De éstas, 12 fueron artrodesis, cinco re-recambios, tres retiros y espaciador solamente y una amputación supracondílea por infección periprotésica grave. De estas 21 re-revisiones, 15 casos fueron de causa séptica (9.6\% del total de recambios). Vale destacar que nueve de las re-revisiones sépticas eran recambios previamente asépti$\cos (60 \%)$. El resto de las reintervenciones (siete) fueron cuatro limpiezas quirúrgicas, una reparación de rotura del aparato extensor, una amputación transfemoral por oclusión arterial aguda (OAA) y una osteosíntesis periprotésica. De las causas asépticas de re-revisión se registraron seis casos (28.6\%), dos casos se debieron a inestabilidad, dos casos por falla de implante, una caso por malalineación y un caso por aflojamiento.

En cuanto a los resultados funcionales, se obtuvieron datos de 98 casos. El KOOS promedio fue de 61.5, siendo 74.9 para la subcategoría síntomas, 72.3 para dolor, 64.0 para AVD, 20 para deporte y recreación y 50.6 para calidad de vida. Se obtuvo un WOMAC promedio de 29.8 (normalizado 68.96) y un SANE de 65. Se registró 32\% de pacientes con un SANE menor o igual a 50.

Se buscó relacionar estadísticamente la causa del recambio con los puntajes funcionales y la supervivencia. Se observó una asociación estadísticamente significativa entre la subcategoría síntomas del KOOS $(\mathrm{p}=0.041)$ y SANE $(\mathrm{p}=$ 0.044 ) en lo que respecta al motivo del recambio, siendo

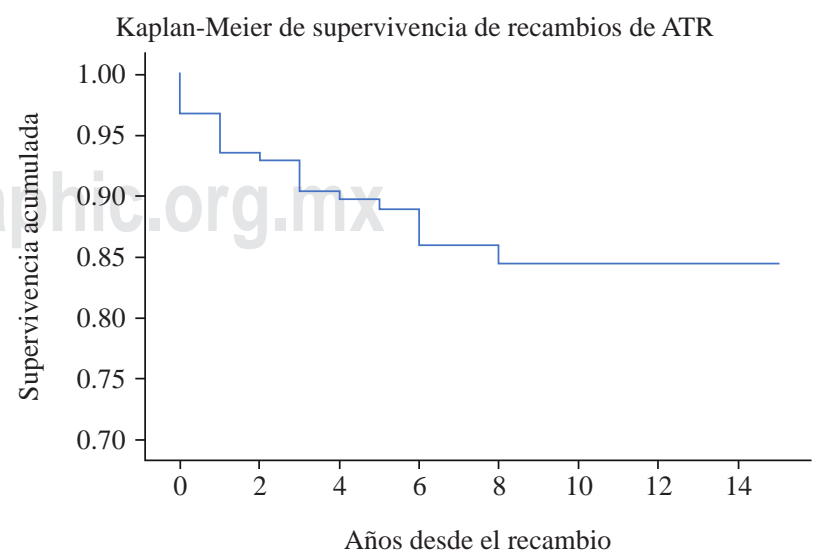

Figura 3: Tabla de supervivencia de recambios de artroplastía total de rodilla. 


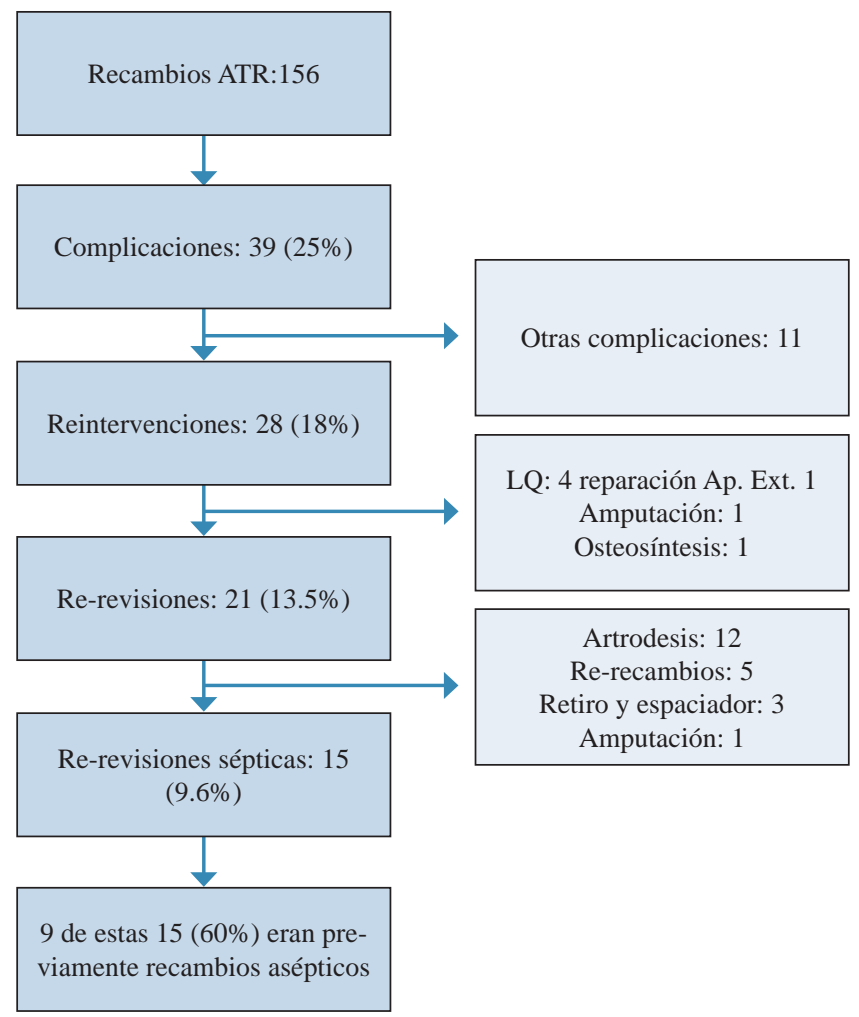

Figura 4: Flujograma de las revisiones. Ap. Extensor $=$ aparato extensor.

mejores los resultados funcionales en casos asépticos frente a sépticos. No se encontró relación estadística entre las variables analizadas y la supervivencia y complicaciones.

\section{Discusión}

El hallazgo más importante de este estudio fue que el porcentaje acumulado de supervivencia de recambio de ATR fue de 93, 89 y 85\% a dos, cinco y 10 años respectivamente, con un promedio de seguimiento de 6.7 años. No obstante, si tomamos en cuenta los fracasos de recambio junto con los fallecidos, destacamos que existe una probabilidad de que solamente $51 \%$ de los recambios serán funcionales a 10 años (paciente vivo sin re-revisión) (Tabla 1). Esto se debe a que la población estudiada es añosa, con un promedio de edad de 71 años y por lo tanto, una tasa de mortalidad más alta que la población general. Pese a que el análisis de supervivencia de Kaplan-Meier es el método más comúnmente utilizado por los registros internacionales, creemos que se deben interpretar estos resultados con precaución, dadas las características de la población estudiada. Es probable que si estudiáramos la supervivencia de los recambios en una población más joven y por ende, con una menor tasa de mortalidad, los resultados de supervivencia serían distintos. Otros estudios previos han mostrado tasas de supervivencia a 10 años de entre 52 y $97 \%$. $^{15,16,17,18,19}$ Según el registro australiano el porcentaje acumulado de re-revisión de ATR a los cinco años fue de 16.6\% ( $\geq 80$ años) y $22.9 \%$ (< 80 años) y a los 10 años 18.3 y $28.1 \%$ respectivamente. Según el registro británico, la tasa acumulada de re-revisión a 10 años fue de $16.62 \% .{ }^{20}$ Otro registro como el neozelandés publicó una supervivencia de $77.80 \%$ a 10 años. ${ }^{21}$ Mientras tanto, Sierra y colaboradores en 2004 encontraron una tasa de supervivencia de $84 \%$ a cinco años $74 \%$ a 10 años y $66 \%$ a 15 años. ${ }^{15,22}$ Sheng y su equipo en 2006 presentaron una supervivencia de $95 \%$ a los dos años, 89\% a cinco años y 79\% a 10 años, mientras Mortazavi en 2011 mostró una tasa de supervivencia de los recambios de causa aséptica de $78 \%$ a ocho años y esta tasa disminuyó a $73 \%$ cuando se incluyeron todas las causas de recambio. ${ }^{16,18}$ Mientras tanto, en un estudio reciente del registro noruego por Leta y colegas presentaron una cohorte de 145 recambios de ATR con una tasa de supervivencia de 85 , 78 y $71 \%$ a los cinco, 10 y 15 años respectivamente. ${ }^{4}$

En algunos estudios se ha observado una asociación entre la supervivencia de los recambios de ATR y el sexo y/o la edad. Nuestro estudio no mostró asociación significativa entre estas variables ( $p=0.311 ; p=0.419$ respectivamente). Según Sheng, Mabry, Suarez y Leta la edad menor de 60 años y el sexo masculino son factores de riesgo de rerevisión. ${ }^{4,16,17,23}$ Sin embargo, Ong y colaboradores no encontraron asociación entre edad y riesgo de re-revisión. ${ }^{24}$ Según Leta y su equipo, el riesgo de re-revisión es el doble en hombres que en mujeres con un riesgo relativo de 2.0. ${ }^{4}$ Otros autores no detectaron diferencias entre sexos, lo cual se puede atribuir a que presentan menor tamaño muestral y una mayor proporción de mujeres en la muestra (178 vs 18). ${ }^{23}$

\section{Características de la población estudiada}

La edad promedio de los pacientes en nuestro estudio a la hora de la cirugía fue de 71 años y $60.9 \%$ de la población fue de sexo femenino. Estas características son similares a las reportadas en otros trabajos. . $^{19,23,25}$

En cuanto al tipo de prótesis utilizada para el recambio y su supervivencia, es difícil encontrar resultados con diferencias estadísticamente significativas, dado que en su gran mayoría fueron de tipo semiconstreñido no abisagrado tipo

\begin{tabular}{|ccc|}
\hline \multicolumn{2}{|c|}{ Tabla 1: Recambios funcionales/total de recambios. } \\
\hline $\begin{array}{c}\text { Número de } \\
\text { recambios }\end{array}$ & $\begin{array}{c}\text { Seguimiento } \\
\text { (años) }\end{array}$ & $\begin{array}{c}\text { Probabilidad de } \\
\text { recambio funcional (\%) }\end{array}$ \\
\hline 156 & 1 & 93.59 [IC: $89.43-97.75]$ \\
156 & 2 & 91.67 [IC: $87.01-96.32]$ \\
156 & 3 & 87.82 [IC: $82.37-93.27]$ \\
156 & 4 & 83.33 [IC: $77.17-89.50]$ \\
127 & 5 & 80.32 [IC: $73.01-87.62$ ] \\
110 & 6 & 73.64 [IC: $64.95-82.33$ ] \\
88 & 7 & 67.05 [IC: $56.66-77.67]$ \\
74 & 8 & 66.22 [IC: $54.73-77.67]$ \\
61 & 9 & 62.30 [IC: $49.31-75.28]$ \\
49 & 10 & 51.02 [IC: $36.00-66.04]$ \\
\hline
\end{tabular}


TC3. Recordamos a su vez que los recambios únicos del componente patelar, como del inserto de polietileno, fueron excluidos de este trabajo. Por otra parte, todas las prótesis fueron cementadas y no hubo casos de prótesis abisagrada.

Según Meijer y colaboradores en una cohorte retrospectiva de 69 recambios de ATR, las principales indicaciones fueron debido a causa séptica en 30\% de los casos, $25 \%$ por aflojamiento aséptico y $25 \%$ desgaste-osteólisis de la artroplastía primaria. ${ }^{26}$ Según el FNR se registró un comportamiento similar, siendo $37.18 \%$ de las indicaciones de recambio por causa séptica.

\section{Características de re-revisiones}

De un total de 156 recambios, hubo 21 re-revisiones, de las cuales 15 fueron de causa séptica (9.6\% del total de recambios). Recordamos que la tasa total de infección, ya sea tratada con antibiótico, limpieza quirúrgica y/o re-revisión fue de $14 \%$. De estas re-revisiones sépticas, $60 \%$ eran previamente asépticas. Esto nos hace pensar si alguno de estos aflojamientos asépticos se debió a una infección a bajo ruido no diagnosticada. Más de la mitad de las re-revisiones se trataron mediante artrodesis (12 casos), de los cuales solamente dos casos fueron re-revisiones asépticas. Mientras tanto, hubo cinco re-recambios en los cuales sólo un caso fue re-revisión de causa séptica.

En cuanto a la causa de fracaso del recambio de ATR, en nuestro estudio se observó un predominio de la causa infecciosa, siendo $71.4 \%$ de las re-revisiones, concordando con otros trabajos similares. ${ }^{4,17,18,19}$ Leta y colaboradores en su cohorte de 145 recambios de ATR, 28\% fracasó por causa séptica. Cabe destacar que en esta serie de pacientes, el motivo de recambio fue de causa aséptica en todos los casos. ${ }^{4}$ En nuestro estudio este porcentaje alcanzó 60\%. Mientras tanto, el resto de los autores citados incluyeron recambios sépticos y asépticos en sus resultados, por lo cual obtuvieron tasas incluso más altas de fracaso de causa séptica de recambios de ATR. Al igual que otros autores, creemos que el riesgo aumentado de fracaso séptico del recambio se debe a la poca vascularización del tejido luego de varias cirugías, el mayor tiempo quirúrgico, implantes más grandes, comorbilidades y el aumento de la edad promedio de estos pacientes. ${ }^{27,28}$

\section{Resultados funcionales}

El principal objetivo de la ATR tanto primaria como del recambio es calmar el dolor, restaurar la función y la movilidad de la rodilla. En nuestro estudio se obtuvo un WOMAC promedio de 29.8 (normalizado 68.96). Ghomrawi y colaboradores en 2009 evaluaron 221 casos de recambios de ATR donde se obtuvo un WOMAC promedio a dos años de seguimiento de 33.1. ${ }^{29}$ Más recientemente Baier y su equipo en 2013 revisaron 78 casos con un seguimiento promedio similar al nuestro (6.75 años), el cual obtuvo un WOMAC promedio de 34 , mostrando también resultados similares a nuestro estudio. ${ }^{30}$ Mientras tanto, otros autores presentaron resultados WOMAC entre 62 y 79 para una escala normalizada de 0 a $100 .^{31,32}$ En cuanto al KOOS, menos trabajos utilizan este puntaje para valorar el resultado funcional de los recambios de ATR. Nosotros obtuvimos un puntaje promedio de 61.5 (síntomas: 74.9; dolor: 72.3; AVD: 64.0; DYR: 20.0 y calidad de vida (CV): 50.6). Mientras tanto, Goldstein y colaboradores presentaron resultados similares según las subcategorías descritas para el KOOS 34. Leta y colegas en otro trabajo en 2016 obtuvieron puntuaciones promedio de KOOS en síntomas 47, dolor 55, AVD 57, DYR 35 y CV 61 en 768 recambios de ATR. La principal diferencia con respecto a nuestro estudio se observa en la subescala de síntomas. ${ }^{33,34} \mathrm{Si}$ bien no encontramos estudios que utilicen el puntaje SANE para recambios de ATR, nosotros obtuvimos un promedio de 65 , siendo menor o igual a 50 en un tercio de los casos. Esto puede significar que pese a que el resto de las puntuaciones funcionales obtenidas son similares a las reportadas en la literatura, la percepción del paciente no es del todo buena.

\section{Limitaciones}

Como limitaciones de este trabajo destacamos que se trata de un estudio retrospectivo, con un nivel de evidencia IV, donde no se pudo realizar un puntaje preoperatorio igual al postoperatorio. A su vez, comparado con otros registros y estudios, presentamos un tamaño muestral pequeño y un seguimiento que podría considerarse como de mediano plazo. Dado que no fue posible realizar una entrevista personal con cada paciente, los puntajes utilizados no fueron objetivos, lo cual puede generar sesgos en los resultados funcionales. Creemos que la principal fortaleza de este trabajo es que se basa en un registro nacional, incluyendo todos los recambios realizados en el país durante el período mencionado. A su vez se trata de un trabajo multicéntrico, de múltiples cirujanos involucrados y analizado por personas ajenas a los equipos quirúrgicos actuantes en cada caso. Por otra parte, destacamos que la pérdida de seguimiento fue menor de $10 \%$ de los casos.

\section{Conclusión}

Sólo la mitad de los recambios realizados según el registro nacional de artroplastías de rodilla permanecieron funcionales a 10 años.

El grado de satisfacción de los pacientes fue bajo en más de un tercio de los casos. Existió un alto porcentaje de complicaciones (25\%), siendo la infección la más frecuente (14\%) y una frecuencia de re-revisión de $13.5 \%$.

\section{Referencias}

1. Carr AJ, Robertsson O, Graves S, et al. Knee replacement. Lancet. 2012; 379(9823): 1331-40. doi: 10.1016/S0140-6736(11)60752-6.

2. Robertsson O, Bizjajeva S, Fenstad AM, et al. Knee arthroplasty in Denmark, Norway and Sweden. Acta Orthop. 2010; 81(1): 82-9. doi: 10.3109/17453671003685442.

3. Robertsson O, Ranstam J, Sundberg M, W-Dahl A, Lidgren L. The swedish knee arthroplasty register. Bone Joint Res. 2014; 3(7): 217-2. doi: 10.1302/2046-3758.37.2000289. 
4. Leta TH, Lygre SHL, Skredderstuen A, Hallan G, Furnes O. Failure of aseptic revision total knee arthroplasties: 145 revision failures from the norwegian arthroplasty register, 1994-2011. Acta Orthop. 2015; 86(1): 48-57. doi: 10.3109/17453674.2014.964097

5. Furnes O, Espehaug B, Lie SA, Vollset SE, Engesaeter LB, Havelin LI. Early failures among 7,174 primary total knee replacements: A follow-up study from the Norwegian Arthroplasty Register 1994-2000. Acta Orthop Scand. 2002; 73(2): 117-29. doi: 10.1080/000164702753671678.

6. http://www.fnr.gub.uy/estadisticas.

7. Kurtz SM, Ong KL, Schmier J, et al. Future clinical and economic impact of revision total hip and knee arthroplasty. J Bone Joint Surg Am. 2007; 89(Suppl 3): 144-51. doi: 10.2106/JBJS.G.00587.

8. Pugliese L. Indicadores de demografía del envejecimiento. Asesor en Segur Soc. 2010.

9. Bryan RS, Rand JA. Revision total knee arthroplasty. Clin Orthop Relat Res. 1982; (170): 116-122. Available in: http://www.ncbi.nlm. nih.gov/pubmed/7127936.

10. Rand JA, Bryan RS. Revision after total knee arthroplasty. Orthop Clin North Am. 1982; 13(1): 201-12. Available in: http://www.ncbi. nlm.nih.gov/pubmed/7063192.

11. Vessely MB, Whaley AL, Harmsen WS, Schleck CD, Berry DJ. The chitranjan ranawat award: long-term survivorship and failure modes of 1000 cemented condylar total knee arthroplasties. Clin Orthop Relat Res. 2006; 452: 28-34. doi: 10.1097/01.blo.0000229356.81749.11.

12. Bellamy N, Buchanan WW, Goldsmith CH, Campbell J, Stitt LW. Validation study of WOMAC: a health status instrument for measuring clinically important patient relevant outcomes to antirheumatic drug therapy in patients with osteoarthritis of the hip or knee. J Rheumatol. 1988; 15(12): 1833-40. Available in: http://www.ncbi.nlm.nih.gov/pubmed/3068365.

13. Roos EM, Toksvig-Larsen S. Knee injury and Osteoarthritis Outcome Score (KOOS) - validation and comparison to the WOMAC in total knee replacement. Heal QualLife Outcomes. 2003; 1: 1477-7525 (Electronic)):17.

14. Anderson AF, Irrgang JJ, Kocher MS, Mann BJ, Harrast JJ, International Knee Documentation Committee. The International Knee Documentation Committee Subjective Knee Evaluation Form: normative data. Am J Sports Med. 2006; 34(1): 128-35. doi: $10.1177 / 0363546505280214$.

15. Sierra RJ, Cooney WP, Pagnano MW, Trousdale RT, Rand JA. Reoperations after 3200 revision TKAs: rates, etiology, and lessons learned. Clin Orthop Relat Res. 2004; (425): 200-6. Available in: http://www.ncbi.nlm.nih.gov/pubmed/15292808.

16. Sheng P-Y, Konttinen L, Lehto M, et al. Revision total knee arthroplasty: 1990 through 2002. A review of the Finnish arthroplasty registry. J Bone Joint Surg Am. 2006; 88(7): 1425-30. doi: 10.2106/ JBJS.E.00737.

17. Suarez J, Griffin W, Springer B, Fehring T, Mason JB, Odum S. Why Do revision knee arthroplasties fail? J Arthroplasty. 2008; 23(Suppl.6): 99-103. doi: 10.1016/j.arth.2008.04.020.

18. Mortazavi SMJ, Molligan J, Austin MS, Purtill JJ, Hozack WJ, Parvizi J. Failure following revision total knee arthroplasty: infection is the major cause. Int Orthop. 2011; 35(8): 1157-64. doi: 10.1007/s00264010-1134-1.

19. Bae DK, Song SJ, Heo DB, Lee SH, Song WJ. Long-term survival rate of implants and modes of failure after revision total knee arthroplasty by a single surgeon. J Arthroplasty. 2013; 28(7): 1130-4. doi: 10.1016/j.arth.2012.08.021.

20. Gujarat Green Revolution Company. National Joint Registry for England, Wales, Northern Ireland and the Isle of Man. 15th Annual Report. Vol 1821, 2017.

21. The New Zealand Joint Registry Annual Report Editorial Committee. 2017.

22. https://aoanjrr.sahmri.com.

23. Mabry TM, Vessely MB, Schleck CD, Harmsen WS, Berry DJ. Revision total knee arthroplasty with modular cemented stems: longterm follow-up. J Arthroplasty. 2007; 22(6 Suppl 2): 100-105. doi: 10.1016/j.arth.2007.03.025.

24. Ong KL, Lau E, Suggs J, Kurtz SM, Manley MT. Risk of subsequent revision after primary and revision total joint arthroplasty. Clin Orthop Relat Res. 2010; 468(11): 3070-76. doi: 10.1007/s11999-010-1399-0.

25. Orthopride Belgian Hip and Knee Arthroplasty Registry Annual Report. 2015.

26. Meijer MF, Reininga IHF, Boerboom AL, Stevens M, Bulstra SK. Poorer survival after a primary implant during revision total knee arthroplasty. Int Orthop. 2013; 37(3): 415-9. doi: 10.1007/s00264-012-1739-7.

27. Hanssen AD, Rand JA. Evaluation and treatment of infection at the site of a total hip or knee arthroplasty. Instr Course Lect. 1999; 48: 111-22. Available in: http://www.ncbi.nlm.nih.gov/ pubmed/10098033

28. Garvin KL, Cordero GX. Infected total knee arthroplasty: diagnosis and treatment. Instr Course Lect. 2008; 57: 305-15. Available in: http://www.ncbi.nlm.nih.gov/pubmed/18399594

29. Ghomrawi HMK, Kane RL, Eberly LE, Bershadsky B, Saleh KJ, North American Knee Arthroplasty Revision (NAKAR) Study Group. Patterns of functional improvement after revision knee arthroplasty. J Bone Joint Surg Am. 2009; 91(12): 2838-45. doi: 10.2106/ JBJS.H.00782.

30. Baier C, Lüring C, Schaumburger J, et al. Assessing patient-oriented results after revision total knee arthroplasty. J Orthop Sci. 2013; 18(6): 955-61. doi: 10.1007/s00776-013-0467-1.

31. Richards CJ, Garbuz DS, Pugh L, Masri BA. Revision total knee arthroplasty. J Arthroplasty. 2011; 26(8): 1299-304. doi: 10.1016/j. arth.2010.12.003.

32. Greidanus NV, Peterson RC, Masri BA, Garbuz DS. Quality of life outcomes in revision versus primary total knee arthroplasty. $J$ Arthroplasty. 2011; 26(4): 615-20. doi: 10.1016/j.arth.2010.04.026.

33. Goldstein WM, Gordon AC, Swope S, Branson J. Rotating platform revision total knee arthroplasty. J Knee Surg. 2012; 25(1): 45-50. Available in: http://www.ncbi.nlm.nih.gov/pubmed/22624247

34. Leta TH, Lygre SHL, Skredderstuen A, et al. Outcomes of unicompartmental knee arthroplasty after aseptic revision to total knee arthroplasty. J Bone Jt Surg. 2016; 98(6): 431-40. doi: 10.2106/ JBJS.O.00499. 